Reference

\section{work on rabies}

\section{G. S. Turner}

The Natural History of Rabies. Edited by George $\mathbf{M}$. Baer. Volume 1: Pp. xvi+454. \$43.00; £21.50. Volume 2 : Pp. $\quad x v i+387 . \$ 36.00 ; £ 18.00$. (Academic: New York and London, September-October 1975.)

THESE two volumes provide a comprehensive central source of information on many facets of this ancient disease. They examine the molecular biology of the virus, the pathogenesis and pathology of rabies, its diagnosis, epidemiology and its prevention in man. The contributors of the 42 chapters have in many cases either been members of, or advisors to, the World Health Organisation (WHO) expert committee on rabies. Readers active in rabies research will recognise many familiar footprints in well trodden ground.

Whether the contents always justify the title of 'natural history' is arguable since this implies a continuing record of natural phenomena. Advances in rabies research seem to be discontinuous - much of the mythology was dispelled by Pasteur but more than 60 years elapsed before the successful application of cell culture methods enabled the virus to be seen for the first time, and also permitted the first reasonably accurate estimate of its size, the physicochemical and biological properties of which are described in part I. Similar methods, described in chapter 9, enabled mechanisms of infection and growth at the cellular level to be examined.

The section on the pathogenesis of rabies recalls arguments concerning the respective roles of neural and haematogenous spread of virus to the central nervous system (CNS). Evidence is also discussed for extraneural replication of virus before and after invasion of the CNS. Latent and abortive rabies is reviewed here as well as the effects of interferon on infection. Contributors remind us, however, that the scanty histological changes observed in infected animal brain, contrast strikingly with the severe clinical signs observed in rabies, and that its pathogenesis remains "a complicated and little understood series of events",

The section on laboratory diagnosis details techniques which are already described elsewhere (see WHO monograph series No. 23, Laboratory Techniques in Rabies). Surprisingly the passive haemagglutination test for rabies antibody does not appear here.
Almost half the second volume is devoted to epidemiology. Individual chapters provide abundant information on rabies in each of many wild and domestic animals in both natural and experimental conditions. Wildlife data concerns several species indigenous only to the new world. No doubt the omission of global epidemiology reflects difficulties in acquiring information from underprivileged countries in which rabies is endemic.

The chapters devoted to the control of rabies in dogs, cats and other domestic animals seem to prefer vaccination to other means, Although vaccination is undeniably effective it is not a control method likely to be favoured in England at the moment. Included in the methods reviewed for the control of wildlife rabies are population reduction, vaccination, the use of gametocides and more recently the use of anticoagulants for destroying vampire bats. The methods mostly suggest directions for future research rather than immediate solution of this intransigent problem.

The final chapters deal with rabies in man, its prevention, first aid treatment, diagnosis and clinical manifestations. Recent optimistic hopes of cure of the overt disease by aggressive intensive care are also mentioned. Here too, details are given of the passive immunisation of man with anti-rabies serum as well as a highly critical review of human, postexposure vaccine therapy and the difficulties of assessing its efficacy. An appendix gives a guide to specific treatment.

Not every contribution is a model of stylistic or verbal excellence and the occasional mistake has escaped the proof reader. This is probably inevitable in a work of this magnitude and in no way detracts from its value as the best and most practical reference text on the subject that has appeared in recent times.

\section{Endocrine system in perspective}

\section{K. Fotherby}

Comparative Vertebrate Endocrino$\operatorname{logy}$. By P. J. Bentley. Pp. xi+415. (Cambridge University: Cambridge, London and New York, January 1976.) Cloth £12; Paperback £4.95.

ENDOCRINOLOGY is a fascinating subject and to those of us who deal mainly with human endocrinology this fascination is increased by taking a broader view and looking at the way in which the endocrine system has evolved. For this purpose this textbook is ideal. It presents the subject matter in a read- able way emphasising the differences between species from a functional aspect. It not only contains a wealth of information concerning the endocrinology of the various species but also contains more than enough basic information concerning the biosynthesis, metabolism and mode of action of the hormones for the book to be read and understood without the necessity of referring to a textbook of basic endocrinology.

In addition to chapters on the comparative morphology of endocrine tissues and the chemical structure and polymorphism and evolution of hormones, there are chapters dealing with the relationship between endocrinology and nutrition, calcium metabolism, the integument, osmoregulation, and reproduction. The text is illustrated with many excellent diagrams.

Although there are species differences in the morphology of the endocrine tissues, and in the chemical structure and biological and immunological behaviour of the hormones, many of these variations are such that they can often be placed into categories corresponding to the major systematic groups of vertebrates. These species differences suggest an orderly evolutionary change. The pathways of hormone synthesis, mechanisms of secretion and mode of action seem to be similar in all vertebrates although the nature of the stimulus responsible for the secretion of a particular hormone may vary according to the physiological role in which the hormone may be involved.

The longest chapter in the book is that devoted to reproduction. This is not surprising since the reproductive process shows a wide diversity of morphological and physiological characteristics, and the latter requires the co-ordination of many different events which are controlled by a number of different hormones. In spite of the wide variation in the morphological and physiological aspects of reproduction, the nature of the hormones involved varies little. The gonadal steroid hormones have the same structure in all vertebrates. More variation is shown by the gonadotrophins; and it is of interest that in most tetrapods the pituitary secretes two gonadotrophins, luteinising hormone and folliclestimulating hormone, whereas in some vertebrates e.g. fishes, only a single gonadotrophin is secreted.

Although written as a textbook for graduate and undergraduate courses in comparative endocrinology, it should be read by all interested in endocrinology. It will be useful as a reference book for research workers, and the availability of a paperback edition may ensure a wide sale for this excellent book. 